
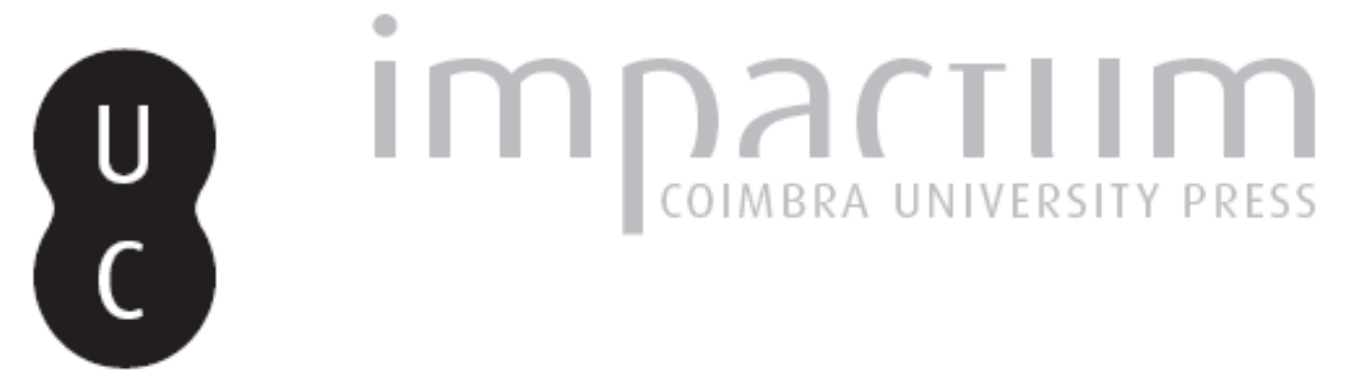

\title{
Plutarque et le politique à la lumière du théâtre Grec
}

Autor(es): $\quad$ Teixeira, Etienne

Publicado por: International Plutarch Society

URL persistente:

URI:http://hdl.handle.net/10316.2/36383

DOI:

DOI:http://dx.doi.org/10.14195/0258-655X_7_8

Accessed : $\quad$ 26-Apr-2023 13:24:13

A navegação consulta e descarregamento dos títulos inseridos nas Bibliotecas Digitais UC Digitalis, UC Pombalina e UC Impactum, pressupõem a aceitação plena e sem reservas dos Termos e Condições de Uso destas Bibliotecas Digitais, disponíveis em https://digitalis.uc.pt/pt-pt/termos.

Conforme exposto nos referidos Termos e Condições de Uso, o descarregamento de títulos de acesso restrito requer uma licença válida de autorização devendo o utilizador aceder ao(s) documento(s) a partir de um endereço de IP da instituição detentora da supramencionada licença.

Ao utilizador é apenas permitido o descarregamento para uso pessoal, pelo que o emprego do(s) título(s) descarregado(s) para outro fim, designadamente comercial, carece de autorização do respetivo autor ou editor da obra.

Na medida em que todas as obras da UC Digitalis se encontram protegidas pelo Código do Direito de Autor e Direitos Conexos e demais legislação aplicável, toda a cópia, parcial ou total, deste documento, nos casos em que é legalmente admitida, deverá conter ou fazer-se acompanhar por este aviso.

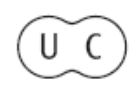




\section{PLOUTARCHOS, n.s.}

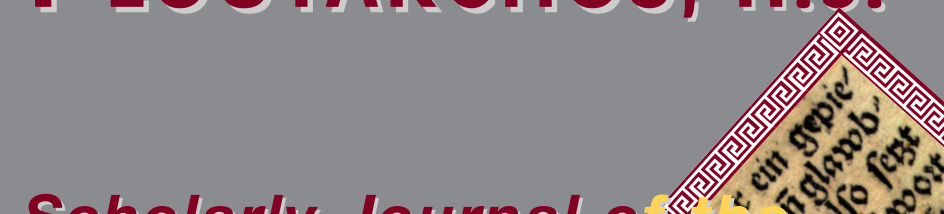

\section{Scholarly Journal o}

International Plut
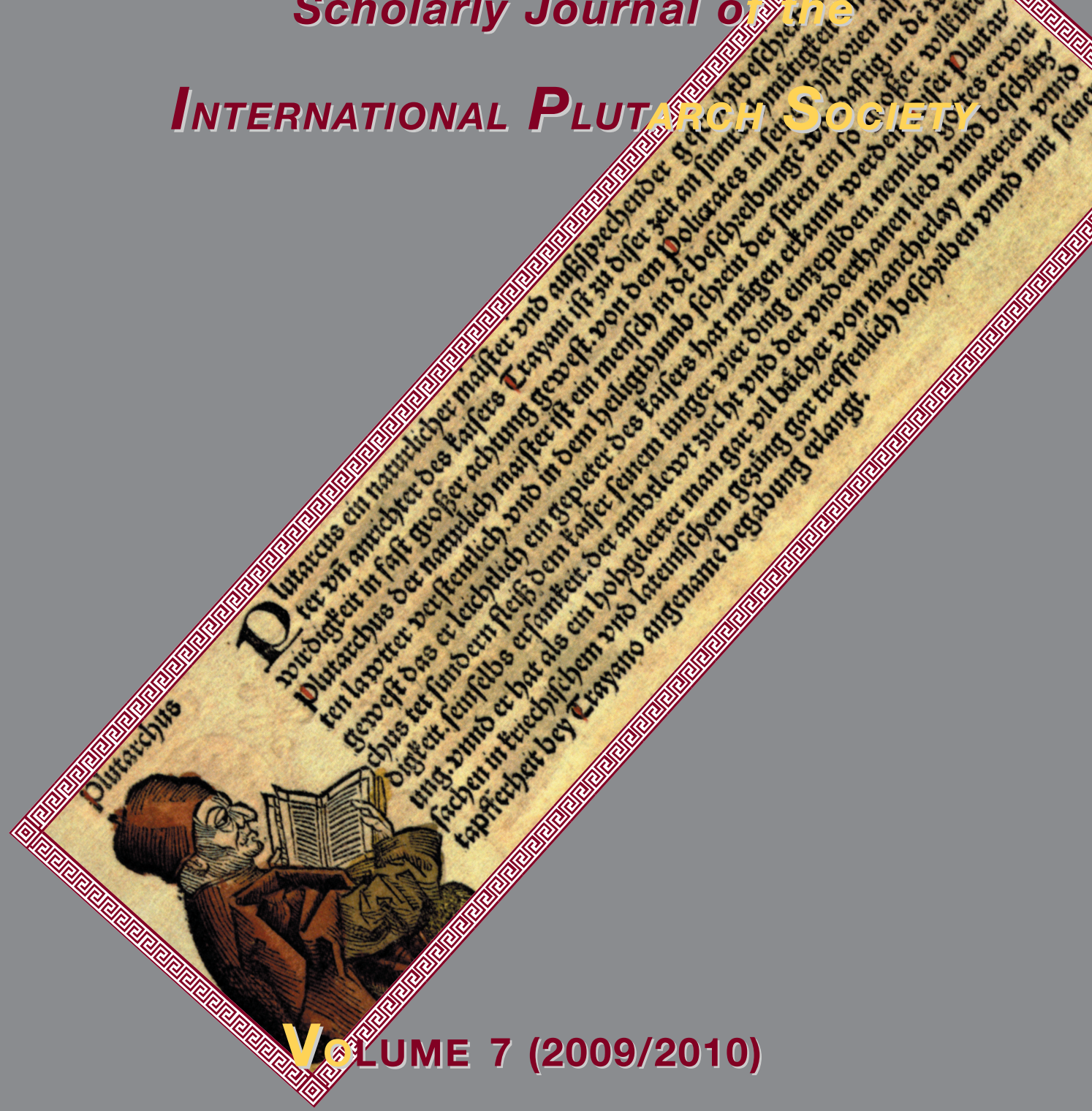

UNIVERSITY OF MÁLAGA (SPAIN) Utah State University, Logan, Utah (U.S.A.) 


\title{
Plutarque et le politique à la lumière du théâtre Grec par \\ Etienne Texeira \\ Université de Dakar \\ eteix2001@yahoo.fr
}

\begin{abstract}
Plutarch has been interested very much in questions concerning politics. He himself has exercised some charges in this domain. This essay is going to discuss about what Plutarch thinks about stastesman through greek theatre.
\end{abstract}

Key-Words: Plutarch, Moralia, Greek Theatre, Greek Politicians.

urieux de tout, Plutarque
toucha à toutes les scien-
ces. C'est ainsi qu'il s'est
intéressé aux questions relatives à la politique ${ }^{1}$, c'est-à-dire à la gestion de la cité. Il a eu lui-même à exercer quelques charges dans ce domaine $^{2}$. La présente étude se propose d'examiner la conception que Plutarque a de l'homme d'Etat, à partir des réflexions que lui a inspirées son commerce avec le théâtre grec.

Quel est le rôle joué par l'éloquence dans la vie de l'homme politique ? Selon Plutarque, il convient tout d'abord de faire preuve de prudence dans ce domaine. C'est ainsi, semble$\mathrm{t}$-il, qu'il faut interpréter le vers d'Euripide que Plutarque cite dans le Praecepta gerendae reipublicae ${ }^{3}$ :

1 Plutarque est l'auteur des traités de politique suivants : Préceptes politiques, De l'exil, Si un vieillard doit s'occuper des affaires publiques, Le philosophe doit s'entretenir avec les pinces, A un prince ingorant, De la monarchie, De la démocratie et de l'aristocratie.

2 Il fut député, jeune encore, par ses concitoyens de Chéronée, auprès du proconsul d'Achaïe, à Corinthe, pour y traiter de leurs intérêts. Plutarque vécut également quelque temps à Rome, où il gagna peu à peu la confiance de Trajan, qui lui confia la direction du jeune Hadrien et ensuite le gouvernement de l'Illyrie.

3 Praec.ger.reip., 803 B. Pour nous conformer à l'usage, nous indiquons en latin les titres des traités politiques. 
Mais s'il s'agit des compositions oratoires d'un Ephorus, d'un Théopompe, d'un Anaximène, s'il s'agit des belles périodes qu'ils débitent en armant leurs soldats et en les rangeant en bataille, il est permis de s'écrier :

«Si près du fer peut-on débiter ces folies $» !^{4}$.

Ainsi, de même que lorsqu'on se sent menacé ou surpris, il arrive que l'on n'ose pas manifester quelque signe de courage que ce soit, de même face à certaines situations il vaut mieux observer un certain silence, voire quelque mutisme.

Il va cependant sans dire que l'homme d'Etat doit se montrer éloquent au moment opportun, au lieu de donner l'impression d'être battu par un adversaire que Plutarque qualifie, empruntant ses termes au vers 137 des Cavaliers d'Aristophane, de :

Rapace braillard, avec une voix de Cycloboros ${ }^{5}$.

L'exagération exprimée dans ce vers ne doit pas nous surprendre, si nous savons qu'Aristophane s'en prend, une fois de plus, à Cléon ${ }^{6}$. Mais peut-être l'auteur du Praecepta gerendae reipublicae, en employant ce vers, veut-il nous faire comprendre qu'il ne sert à rien de recourir à une éloquence excessive, et que, si par les discours l'homme politique parvient à persuader son peuple, on oublie assez facilement que le caractère de l'individu peut jouer un rôle important dans la persuasion. Aussi Plutarque propose-t-il de rectifier le vers de Ménandre :

$C$ 'est non par ses discours, mais par son caractère

Qu'on persuade un peuple et que l'on sait lui plaire

en reconnaissant que la persuasion s'exerce à la fois par le caractère et par les discours ${ }^{7}$.

La puissance de séduction d'Alcibiade est légendaire. Cependant, Plutarque semble se demander si, sur le plan de l'éloquence, il jouissait du succès auquel il était en droit de s'attendre ${ }^{8}$, car, comme le dit le poète comique Eupolis, il était

Un causeur excellent, un médiocre orateur ${ }^{9}$.

Plutarque, on le voit, trouve comme par hasard, serions-nous tenté de dire, le vers approprié pour caractériser l'éloquence d'Alcibiade.

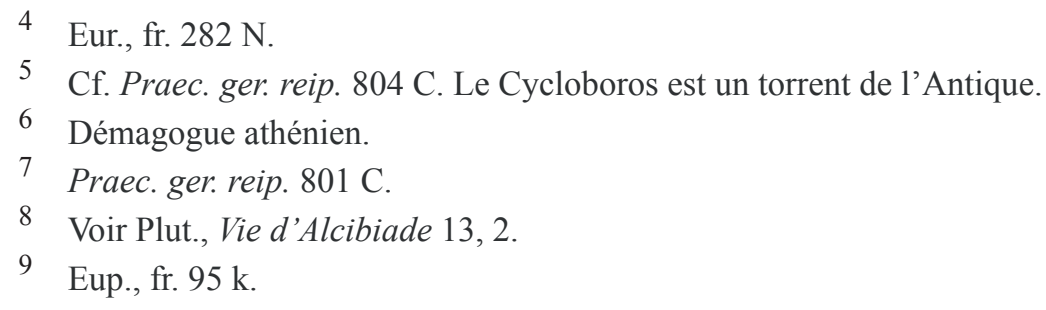


Quant à Cinéas, Thessalien qui passait pour très avisé et loquace ${ }^{10}$, il semble qu'il ait mis à profit son éloquence pour s'acquitter honorablement des missions que lui confiait Pyrrhus dans les villes, au point que, selon Plutarque ${ }^{11}$, il justifiait la maxime d'Euripide :

\section{La parole écarte tout obstacle}

Que pourrait opposer le fer des ennemis ${ }^{12}$.

Ces vers, prononcés par Etéole s'adressant à Jocaste, affirment, comme le fait remarquer V. Di Benedetto, les capacités exceptionnelles du logos qui arrive à l'emporter sur les armes ${ }^{13}$. C'est dire qu'en politique les différends ne se règlent pas systématiquement par le fer, autrement dit par la violence.

Peu commune également était 1'éloquence de Lucullus, qui n'était pas appropriée uniquement aux affaires, à la manière des autres orateurs qui se démènent sur le forum

Comme un thon harponné se débat dans les flots ${ }^{14}$,

et dont la faconde, hors du forum, se dessèche et languit par manque de culture ${ }^{15}$.

L'image du thon qui se débat, que Plutarque emprunte à un poète tragique inconnu, traduit bien l'embarras dans lequel peuvent se trouver certains orateurs, mais auquel Lucullus, grâce à son éloquence, essentiellement, a su échapper la plupart du temps.

Si la puissance de séduction d'Alcibiade, disions-nous plus haut, est légendaire, l'éloquence de Périclès ne l'est pas moins. C'est ainsi que Plutarque nous apprend que son surnom d'Olympien lui vint surtout de son éloquence, car, selon les poètes de son temps, lorsqu'il parlait au peuple, « il tonnait, il lançait des éclairs » et « sur sa langue il portait un foudre redoutable ${ }^{16}$. On peut donc dire que si Zeus a pour attribut le foudre, Périclès a pour arme son éloquence. C'est avec la parole qu'il lance les éclairs, qu'il tonne et que la Grèce est ébranlée. Il est donc, pour reprendre le mot, déjà ancien, de A. Couat, « la langue la plus puissante de toute la Grèce » ${ }^{17}$.

10 Il avait suivi les leçons de l'orateur Démosthène.

11 Vie de Pyrrhus 14,2.

12 Eur., Phéniciennes 516-517.

13 V. Di Benedetto, Euripide : teatro e società, Torino, 1971, p. 203.

14 Plut., Vie de Lucullus 1, 5.

15 Fr. trag. ades. 387 Nauck $^{2}$.

16 Vie de Périclès 8, 4. Plutarque pense sans aucun doute aux vers 530-531 des Acharniens d'Aristophane.

17 A. CouAt, Aristophane et l'ancienne comédie attique, Paris, 1889, p. 133. 
Ainsi, l'importance de l'éloquence, en politique, est indéniable. Voyons, à présent, quelle est la place du vieillard dans la politique ${ }^{18}$.

Le vieillard peut-il participer à la vie politique ou doit-on l'en écarter systématiquement ? En citant, dans le An seni sit gerenda respublica ${ }^{19}$, les propos du soldat dans la comédie :

Mes cheveux blancs font que désormais on ne me donne plus de solde 20

Plutarque compare, implicitement certes, ce vieux soldat au vieil homme d'Etat, mais il faut admettre que l'un et l'autre, dans la mesure où ils continuent d'être au service de la patrie, doivent jouir pratiquement des mêmes droits que leurs cadets, d'autant que l'on se fie parfois davantage à l'efficacité de beaucoup de vieillards qu'à celle de certains jeunes.

Dès lors, il est normal que le vieil homme d'Etat encore actif soit entouré de considération et de respect, car l'imagination dont il est capable de faire preuve,

lorsqu'elle est en activité, luit comme un bronze magnifique, pour reprendre, avec Plutarque, les mots de Sophocle ${ }^{21}$.

C'est pourquoi il n'y a pas lieu de décourager le vieillard qui désire continuer à participer, d'une manière ou d'une autre, à la vie politique, d'autant que son concours peut être, bien entendu, d'une utilité fort appréciable, vu sa longue expérience en la matière. Au demeurant, Euripide n'écrit-il pas :

Un sage et bon conseil veut mieux que mille bras ${ }^{22}$ ?

Développant cette maxime, Plutarque précise :

Un seul avis, dicté par la prudence et la persuasion, assure dans les affaires publiques les plus beaux et les plus importants résultats $^{23}$.

Il faut donc voir dans cette citation une marque de sagesse d'Euripide luimême.

Les vers 268-269 de l'Héraclès d'Euripide :

Ô mon bras, comme tu désires saisir la lance,

Mais ton désir se consume dans l'impuissance!

Cette question a préoccupé Plutarque au point qu'il a écrit le traité An seni sit gerenda respublica.

An seni 789 C.

20 Adesp. fr. $226 \mathrm{~K}$.

21 Soph., fr. $780 \mathrm{~N}$.

22 Eur., fr. $200 \mathrm{~N}$.

23 An seni 790 A (trad. V. Bétolaud). 
Sont cités par Plutarque ${ }^{24}$ pour signifier que le bon sens le plus élémentaire admet l'incapacité du vieillard à mener des activités politiques aussi intenses qu'auparavant, encore que pour celui qui ne peut rester inactif il existe des tâches qui réclament un « doux effort » et entraîne une « fatigue agréable à subir ${ }^{25} »$.

Les vieux hommes d'Etat peuvent certes beaucoup apporter à la vie politique de leur cité, mais encore fautil qu'ils aient le courage et la force nécessaire leur permettant de continuer à s'y intéresser.

Hormis cette catégorie d'hommes politiques qui, vu leur longue expérience, donnent généralement satisfaction plus qu'ils ne déçoivent, l'examen de certaines citations nous amène à poser le problème de l'existence de l'homme politique idéal.

Il convient tout d'abord de noter que Plutarque s'inspire de l'exemple du protagoniste qui, au théâtre, se soumet à un acteur d'occasion qui ne joue qu'un rôle secondaire et qui lui parle avec déférence, puisque ce dernier porte sceptre et diadème, pour évoquer le mépris que le riche dans sa magnificence éprouve pour le magistrat simple et pauvre ${ }^{26}$. Une telle attitude est assurément indigne du riche qui méconnaît l'importance du rôle que peut jouer le magistrat dans la vie de la cité. En clair, le riche semble oublier qu'au théâtre comme dans la vie de tous les jours chacun doit s'acquitter de la tâche qui est assignée, l'essentiel étant qu'il le fasse honorablement.

Le problème étant ainsi posé dans un cadre assez général, Plutarque aborde le cas précis de l'homme d'Etat.

Nous avons examiné plus haut le rôle du vieillard dans la vie politique. Revenons-y brièvement, dans le cadre de la question qui nous occupe, pour souligner que Plutarque, en rapportant dans le An seni sit gerenda respublica ${ }^{27}$ la question posée par Antigone à Edipe :

$$
\begin{aligned}
& \text { Qu'est devenu l'Edipe aux } \\
& \text { glorieuses énigmes }{ }^{28} \text { ? }
\end{aligned}
$$

semble dire que le vieillard qui entre dans la vie politique sans expérience n'est pas meilleur que ne l'était CEdipe, en dépit de sa fameuse solution de l'énigme du sphinx, quand il fut exposé aux vicissitudes de l'exil. Il va donc de soi qu'on ne s'improvise pas homme politique, mais qu'un minimum d'expérience est in-

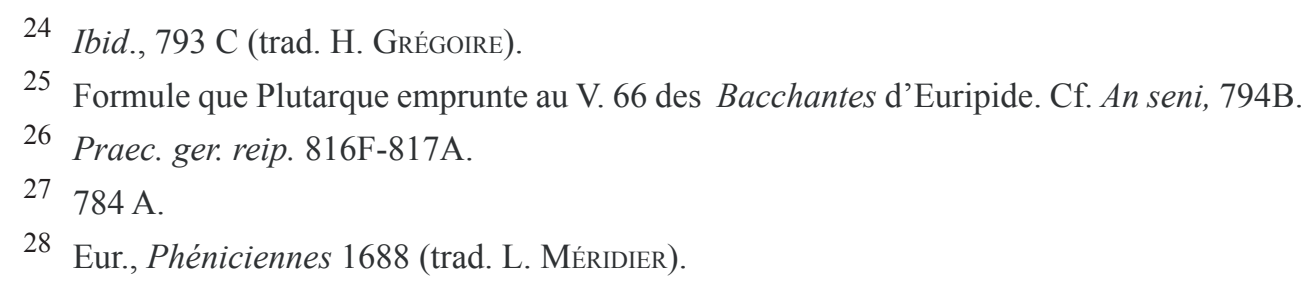


dispensable à celui qui veut mener à bien les affaires de la cité.

Il est également évident que l'homme d'Etat qui veut se faire respecter doit posséder un certain nombre de qualités fondamentales. Cela dit, Plutarque est, bien entendu, conscient que certains défauts peuvent ternir la réputation du politique.

Ainsi, la raison ne devrait pas faire défaut à l'homme d'Etat; elle doit guider ses choix ainsi que les décisions qu'il pourrait être amené à prendre. Pour illustrer l'exemple du châtiment qui devance parfois l'accusation, ou celui du supplice qui frappe avant que le crime ait été démontré, Plutarque cite les vers suivants d'un tragique inconnu :

De maîtriser ses flots leur âme est incapable :

C'est l'ancre dont la pointe a mordu dans le sable 29 .

Et Plutarque de commenter :

Ils (les hommes d'Etat) n'y parviendront point, si la raison par la gravité de ses enseignements ne terrasse, n'écrase l'autorité absolue. Le souverain doit imiter le soleil qui, parvenu à sa plus grande hauteur et s'étant élevé dans les signes septentrionaux, éprouve à peine le moindre mouvement. La lenteur de sa marche en garantit la sûreté ${ }^{30}$.

Le sens des responsabilités à partager ne doit pas échapper à l'homme d'Etat. A quoi sert, en effet, de vouloir tout accaparer, au risque de ne pas s'acquitter comme il faut de sa tâche? Le politique ressemble alors à ce soidisant menuisier qui fait un travail ne relevant pas de la menuiserie ${ }^{31}$.

Par ailleurs, l'exemple d'Agésilas qui, lorsqu'il était sérieusement pressé par ses amis, perdait toute sa force et toute sa dignité ${ }^{32}$, n'est pas à imiter. Plutarque compare cette attitude à celle du Pégase d'Euripide qui

Se baissait, par frayeur, plus qu'on ne le voulait ${ }^{33}$.

Une certaine fermeté est donc nécessaire, mais cela ne doit pas empêcher l'homme d'Etat de faire preuve de quelque souplesse, s'il veut amener le peuple à obéir plus facilement et plus spontanément, et donc l'empêcher d'opposer un esprit de révolte. C'est à un tragique inconnu que Plutarque emprunte à la citation servant à illustrer cette idée :

Adesp. fr. 379 N.

30 Ad principem indoctum 782 D-E (trad. V. BÉTOLAud).

31 C'est ce que Plutarque signifie dans Eur., fr. 988 N, cité en Praec. ger. reip. 812 E.

32 Praec. ger. reip. $807 \mathrm{E}$.

33 Eur., fr. $309 \mathrm{~N}$. 
Du gouvernail il faut détendre un peu la barre

Quand les flots s'agitent beaucoup ${ }^{34}$.

Cette nouvelle image tirée de la navigation, Plutarque l'explique en écrivant :

Tantôt on usera d'indulgence, tantôt on se prêtera de bonne grâce aux plaisirs du peuple par des sacrifices, des combats publics, des représentations théâtrales ; tantôt, comme il arrive dans les familles pour les fautes des jeunes, on fera semblant de ne pas voir et de ne pas entendre. De cette manière le droit de réprimander et de parler avec franchise conservera toute sa vigueur, comme un remède puissant ${ }^{35}$.

Mais parler avec franchise ne signifie pas utiliser des propos abusifs. Autrement dit, il faut savoir faire la part des choses, à l'instar du Nestor de Sophocle qui, lorsqu'il fut outragé par Ajax, fit, selon Plutarque ${ }^{36}$, une réplique digne de celle d'un homme d'Etat :

Je ne te blâme point : faisant bien, tu dis mal $^{37}$.

34 Adesp. fr. $413 \mathrm{~N}$.

35 Praec. ger. reip. 818 A (trad. V. BÉTOLAUd).

36 Ibid. $810 \mathrm{C}$.

37 Soph., fr. $771 \mathrm{~N}$ (trad. V. BÉtolaud).

38 Cf. Praec. ger. reip. 811 F.

39 Adesp. fr. $1325 \mathrm{~K}$. Trad. V. BÉTOLAud légèrement modifiée.

40 Eur., fr. 974 N (trad. J. Dumortier). Cf. Praec. ger. reip., 811 D.
Un poète comique inconnu, voulant railler Métiochus, un compagnon de Périclès qui semble avoir usé du pouvoir de telle sorte qu'il a soulevé la haine et le dégoût ${ }^{38}$, écrit :

Métiochus, vois-tu, est général, Métiochus inspecte les routes,

Métiochus inspecte le pain, Métiochus surveille le meunier,

Métiochus prend soin de toutes choses, mais gare un jour aux mécomptes ${ }^{39}$ !

Plutôt que de s'approprier toutes les tâches de la cité, l'homme d'Etat doit se comporter de telle sorte qu'il trouve des gens qui l'estiment quand il va vers eux et qui le réclament lorsqu'il n'est pas là.

Quelle solution convient-il que l'homme d'Etat adopte pour satisfaire le peuple ? La réponse, Plutarque l'extrait d'une citation d'Euripide :

Le dieu s'intéresse aux choses d'importance

Mais abandonne au sort des vétilles 40 . 
Ainsi, le politique se doit de se préoccuper d'abord des problèmes qui revêtent une importance capitale; mais cela ne veut pas dire qu'il faille négliger le reste. L'essentiel est qu'il s'acquitte honorablement de son devoir.

Pour conclure, évoquons le régime politique qui, selon Plutarque, convient le mieux à l'homme d'Etat idéal - si tant est que celui-ci puisse exister -, c'est-à-dire la monarchie. Une citation faite par Démétrios Poliorcète, et que Plutarque se plaît à rapporter, illustre bien ce point de vue. L'auteur écrit en effet que l'homme d'Etat qui ne choisit pas la monarchie est

poussé à dire tout haut les mots d'Eschyle que Démétrios Poliorcète employa contre la fortune après avoir perdu son royaume :

$C$ 'est toi qui m'élevas, c'est toi qui me renverses 41 .

C'est dire que pour Plutarque la monarchie peut garantir à l'homme d'Etat une certaine assurance, et au régime une certaine stabilité, contrairement, par exemple, à la démocratie.
Et pour étayer ce point de vue, c'est à Platon le comique que Plutarque emprunte les vers lorsqu'il écrit ${ }^{42}$ :

Ceux qui vivent sous un régime démocratique, par corruption ou par dédain, ou faute d'avoir de meilleurs chefs, acceptent les services des premiers venus, bien qu'ils les détestent et les méprisent ; et puis après ils aiment à entendre dire sur leur compte ce que Platon, l'auteur comique, met dans la bouche du peuple lui-même :

Prends ma main, prends, te dis-je, et cela sans tarder: $\mathrm{ral}^{43}$.

Je vais élire Agyrrhius géné-

Dans un autre endroit, le peuple demande une cuvette, une plume garnie de ses barbes pour se faire vomir, et il dit:

$$
\begin{aligned}
& \text { C'est que Mantias occupe } \\
& \text { ma tribune }{ }^{44} \text {. }
\end{aligned}
$$

Et ailleurs :

Quoi ! nourrir le puant Céphalus! Quel fléau ${ }^{45}$ !

Ces citations traduisent clairement l'aversion du peuple pour certains de ses

41 Esch. fr. 359 N. Cité en De unius in republica dominatione, polulari statu, et paucorum imperio $827 \mathrm{C}$.

Praec. ger. reip. 801 B. Trad. V. BÉTolaud, avec quelques modifications.

43

On lit chez Aristophane, Ploutos 176 (trad. H. VAN DAELE) : « Et Agyrrhius, n'est-ce pas à cause de lui qu'il fait le péteux? " Agyrrhius est un riche démagogue, auteur du triobole, et insolemment fier de sa popularité acquise par ce moyen.

44 V. BÉTolaud traduit : «C'est qu'un vrai pot de chambre occupe la tribune » (sic).

45 Adesp. fr. $185 \mathrm{~K}$. 
dirigeants qui, ayant bénéficié, au début, de sa confiance, finissent par le décevoir ${ }^{46}$.

\section{BibLIOGRAPHIE}

EURIPIDE,

- Les Phéniciennes, Les Bacchantes, Paris, Belles Lettres, 1961

Plutarque,

- Euvres morales, tome XI, $1^{\text {èr }}$ partie, Paris, Belles Lettres, 1984.

- Vies, tome III, Paris, Belles Lettres, 1964.

- Vies, tome VI, Paris, Belles Lettres, 1971.

Bellessort, A.,

- Athènes et son théâtre, Paris, Perrin, 1934.

BENEDETTO, V. DI,

- Euripide : teatro e società, Torino, 1971.
Counat, A.,

- Aristophane et l'ancienne comédie attique, Paris, Lecène-Oudin, 1889.

DenIs, J.,

- La comédie grecque, Paris, Hachette, 1886.

Goossens, R.,

- Euripide et Athènes, Bruxelles, 1962.

NAVARRE, O.,

- Le théâtre grec, Paris, Hachette, 1925.

RoMiLLY, J. DE,

- Problèmes de la démocratie grecque, Paris, Belles Lettres, 1975.

SÉCHAN, L.,

- Etudes sur la tragédie grecque, $2^{\mathrm{e}}$ éd., Paris, Belles Lettres, 1967.

46 Dans son ouvrage intitulé Problèmes de la démocratie grecque, Paris, 1975, p. 122, J. DE Romilly présente la démocratie comme «une politeia dans laquelle l'intérêt de tous est sacrifié à celui d'une faction ». 